cited unanswered questions argue for a cautious approach to vaccination policy until trials have been completed and fully reported.

George F. Sawaya, M.D.

Karen Smith-McCune, M.D., Ph.D.

University of California at San Francisco

San Francisco, CA 94118

1. Joura EA, Leodolter S, Hernandez-Avila M, et al. Efficacy of a quadrivalent prophylactic human papillomavirus (types 6, 11, 16 , and 18) L1 virus-like-particle vaccine against high-grade vulval and vaginal lesions: a combined analysis of three randomised clinical trials. Lancet 2007;369:1693-702.

2. Cancer of the vulva. Bethesda, MD: National Cancer Institute, 2007. (Accessed August 23, 2007, at http://seer.cancer.gov/ statfacts/html/vulva.html.)

DRS. AGOSTI AND GOLDIE REPLY: As we noted, the need for long-term follow-up data are important, which Suba and Raab emphasize. Furthermore, it is imperative that momentum behind efforts in the past decade to develop feasible options for cervicalcancer screening in low-resource settings continues to build. The Bill and Melinda Gates Foundation provided $\$ 55.6$ million to the Alliance for Cervical Cancer Prevention to promote screening and \$13 million toward the development of lowcost HPV DNA tests and other tests. We believe it is inequitable to exclude women in developing countries from the potential benefits of vaccination, new technology, and screening approaches that appear to be promising. ${ }^{1}$

We emphasized that an integrated approach that includes screening and vaccination is likely to prevent the greatest number of deaths from cervical cancer. However, countries will make their own decisions about the best strategic approach to cervical-cancer prevention, accounting for local epidemiologic factors and disease burden, ${ }^{2}$ competing priorities, and the cost-effectiveness, affordability, and feasibility of vaccination programs targeting adolescents and the screening of adult women. We urge that attention be given to realworld solutions for preventing death from cervical cancer in women living in poverty.

Jan M. Agosti, M.D.

Bill and Melinda Gates Foundation

Seattle, WA 98102

Sue J. Goldie, M.D., M.P.H.

Harvard School of Public Health

Boston, MA 02115

1. Sankaranarayanan R, Esmy PO, Rajkumar R, et al. Effect of visual screening on cervical cancer incidence and mortality in Tamil Nadu, India: a cluster-randomized trial. Lancet 2007;370:398-406. 2. World Health Organization. WHO/ICO (Institut Català d'Oncologia) Information Centre on HPV and cervical cancer. (Accessed August 23, 2007, at http://www.who.int/hpvcentre.)

\title{
Human Papillomavirus and Oropharyngeal Cancer
}

TO THE EDITOR: The study by D'Souza et al. (May 10 issue) ${ }^{1}$ on oropharyngeal squamous-cell carcinomas associated with human papillomavirus (HPV) provides important epidemiologic insights into a cancer that is becoming increasingly common in the United States. ${ }^{2}$ However, the molecular mechanisms of carcinogenesis in HPV-associated oropharyngeal squamous-cell carcinomas remain unclear.

The integration of HPV type 16 (HPV-16) into the host genome is an important mechanism in cervical carcinogenesis, ${ }^{3}$ but there is no direct evidence that this process occurs in oropharyngeal squamous-cell carcinomas. The authors state that Southern blot, real-time polymerase-chain-reaction (PCR), and fluorescence in situ hybridization analyses ${ }^{4}$ have established integration sites but that these methods provide only indirect evidence. Direct evidence would require observation of the viral DNA sequence either flanked or attached to one end of human DNA (junction sequences). Mellin et al..$^{5}$ did not observe this finding in HPV-16positive tonsillar carcinomas. We previously used restriction-site PCR in more than 100 HPV-16 and HPV-18 cervical cancers to identify many of these junction sequences. ${ }^{6}$ However, when we used this same technique in 40 oropharyngeal squamouscell carcinomas that were positive for HPV-16, we did not detect junction sequences (unpublished data). This finding, which suggests a mechanism of carcinogenesis that is distinct from that in cervical cancer, warrants further investigation.

Odey C. Ukpo, M.S.

Eric J. Moore, M.D.

David I. Smith, Ph.D.

Mayo Clinic

Rochester, MN 55905

oukpo1979@gmail.com

1. D’Souza G, Kreimer AR, Viscidi R, et al. Case-control study of human papillomavirus and oropharyngeal cancer. N Engl J Med 2007;356:1944-56. 
2. Frisch $M$, Hjalgrim $H$, Jaeger $A B$, Biggar RJ. Changing patterns of tonsillar squamous cell carcinoma in the United States. Cancer Causes Control 2000;11:489-95.

3. Park JS, Hwang ES, Park SN, et al. Physical status and expression of HPV genes in cervical cancers. Gynecol Oncol 1997;65: 121-9.

4. Gillison ML. Human papillomavirus-associated head and neck cancer is a distinct epidemiologic, clinical, and molecular entity. Semin Oncol 2004;31:744-54.

5. Mellin H, Dahlgren L, Munck-Wikland E, et al. Human papillomavirus type 16 is episomal and a high viral load may be correlated to better prognosis in tonsillar cancer. Int J Cancer 2002; 102:152-8.

6. Thorland EC, Myers SL, Gostout BS, Smith DI. Common fragile sites are preferential targets for HPV16 integrations in cervical tumors. Oncogene 2003;22:1225-37.

TO THE EDITOR: Little is known about the natural history of oral HPV infection. ${ }^{1}$ In a cohort of 360 healthy students (mean age, 18.7 years), 69\% of whom were female, ${ }^{2}$ we tested oral cytobrush samples for HPV DNA by means of multiplex PCR. Of these students, 20 (5.6\%) were positive for HPV. Three years later, 8 of 183 students who were retested (4.4\%) were positive, and 1 had persistent infection. Oral HPV infection was unusual, and the persistence of infection was rare.

Of the 183 students who were retested, 28 were sexually inactive, and all these students were HPVnegative. Of the sexually active students, $100 \%$ of those who were HPV-positive had had both penetrative and oral-genital sex in the previous 3 years; of those sexually active students who were HPVnegative, $88 \%$ had had only penetrative sex and $86 \%$ had had only oral-genital sex. These findings support the hypothesis that oral HPV is transmitted through sexual contact and that oral-genital contact is the likely mechanism.

Hilary Williams, M.B., Ch.B., Ph.D.

Bristol Haematology Oncology Centre

Bristol BS2 8ED, United Kingdom

hilary.williams@ubht.nhs.uk

Craig D. Higgins, M.Sc.

London School of Hygiene and Tropical Medicine

London WCIE 7HT, United Kingdom

Dorothy H. Crawford, M.D., Ph.D.

University of Edinburgh

Edinburgh EH9 1QH, United Kingdom

1. Syrjänen S. Human papillomaviruses in head and neck carcinomas. N Engl J Med 2007;356:1993-5. [Erratum, N Engl J Med 2007;357:313.]

2. Higgins CD, Swerdlow AJ, Macsween KF, et al. A study of risk factors for acquisition of Epstein-Barr virus and its subtypes. J Infect Dis 2007;195:474-82.

TO THE EDITOR: D'Souza et al. report a very high prevalence of HPV involving $72 \%$ of oropharyngeal squamous-cell carcinomas. This finding is unlikely to be related to the detection method, ${ }^{1}$ since in situ hybridization was used, a reliable technique with a test outcome that is often similar to that of viral oncogene transcript analysis. ${ }^{2}$ We previously reported that in a Dutch cohort, 6 of 37 oropharyngeal carcinomas (16\%) contained transcriptionally active HPV. ${ }^{3}$ This prevalence differs significantly $(\mathrm{P}<0.001)$ from that reported by D'Souza et al. A review article also reported a prevalence of HPV in oropharyngeal carcinomas that was much lower than $72 \% .{ }^{4}$ We wonder whether the associations reported by DeSouza et al. can be extrapolated to other populations.

Boudewijn J. Braakhuis, Ph.D.

Peter J. Snijders, Ph.D.

C. René Leemans, M.D., Ph.D.

VU University Medical Center

1081 HV Amsterdam, the Netherlands

bjm.braakhuis@vumc.nl

1. Syrjänen S. Human papillomaviruses in head and neck carcinomas. N Engl J Med 2007;356:1993-5. [Erratum, N Engl J Med 2007;357:313.]

2. Smeets SJ, Hesselink AT, Speel E-JM et al. A novel algorithm for reliable detection of human papillomavirus in paraffin embedded head and neck cancer specimen. Int J Cancer (in press).

3. Braakhuis BJM, Snijders PJF, Keune W-JH, et al. Genetic patterns in head and neck cancers that contain or lack transcriptionally active human papillomavirus. J Natl Cancer Inst 2004; 96:998-1006.

4. Kreimer AR, Clifford GM, Boyle P, Franceschi S. Human papillomavirus types in head and neck squamous cell carcinomas worldwide: a systematic review. Cancer Epidemiol Biomarkers Prev 2005;14:467-75.

THE AUTHOR REPLIES: The results of in situ hybridization correlate with viral oncogene expression in our laboratory (unpublished data). Therefore, it is unlikely that the high prevalence of HPV in oropharyngeal cancers that we found can be explained by false positive misclassification. However, our study was performed in a hospital and was not population-based. We cannot exclude the possibility that subjects who did not have traditional risk factors were more likely to participate in the study. The HPV prevalence of $72 \%$ was similar to the $63 \%$ prevalence in cancers of the oropharynx that were collected throughout the United States in a clinical trial conducted by the Eastern Cooperative Oncology Group. ${ }^{1}$

We acknowledge that the fraction of oropharyngeal cancers caused by HPV in the United States may differ from that in other geographic regions. Cross-sectional prevalence in a population would largely be driven by incidence rates for HPV-positive and HPV-negative squamous-cell cancers of 
the head and neck. One might reasonably expect that the relative incidence of these two cancers would be driven by local societal mores - for example, the prevalence of alcohol and tobacco use, sexual behaviors, and other cofactors (including diet and oral hygiene) in a population. Incidence rates may also be quite dynamic, because behaviors may change considerably over time. For instance, a significant increase in the prevalence of HPV-associated tonsillar cancer from about 23\% in the 1970 s to $68 \%$ in the period from 2000 through 2002 was reported in Sweden. ${ }^{2}$ Therefore, geographic variation in the prevalence of HPV in oropharyngeal cancers may be strongly influenced by the region and calendar period sampled.

Although viral integration occurs in the majority of cervical cancers, it is neither necessary for nor specific to invasive carcinoma. ${ }^{3}$ Increased expression and stability of viral oncogene transcripts occur as a consequence of viral integration. Analogous deregulation of viral oncogene expression may occur in episomal virus through methylation or mutation of the viral upstream regulatory region. ${ }^{3}$ Although we agree with Ukpo et al. that patterns of in situ hybridization and RT-PCR are indirect measures of integration, analysis of restriction-fragment-length polymorphisms by Southern blot hybridization is a direct measure. Viral integration into the genome of head-and-neck squa- mous-cell carcinoma has been demonstrated by this method ${ }^{4}$ and through the cloning of viral-cell genome fusion sites, ${ }^{5}$ albeit in few cases.

Although oral HPV infection is now recognized as a causative factor for a subgroup of head-andneck squamous-cell carcinomas, little is known about the natural history of oral HPV infection. Natural-history studies are needed to gain a better understanding of the risk factors for acquisition of oral HPV infection and the factors that affect the duration of infection.

Maura L. Gillison, M.D., Ph.D.

Johns Hopkins Medical Institutions Baltimore, MD 21231

gillima@jhmi.edu

1. Fakhry C, Westra WH, Li S, et al. Prognostic significance of human papillomavirus (HPV) tumor status for patients with head and neck squamous cell carcinoma (HNSCC) in a prospective, multi-center phase II clinical trial. J Clin Oncol 2007; Suppl:25. abstract.

2. Hammarstedt L, Lindquist D, Dahlstrand H, et al. Human papillomavirus as a risk factor for the increase in incidence of tonsillar cancer. Int J Cancer 2006;119:2620-3.

3. Pett $M$, Coleman N. Integration of high-risk human papillomavirus: a key event in cervical carcinogenesis? J Pathol 2007; 212:356-67.

4. Steenbergen RD, Hermsen MA, Walboomers JM, et al. Integrated human papillomavirus type 16 and loss of heterozygosity at 11q22 and 18q21 in an oral carcinoma and its derivative cell line. Cancer Res 1995;55:5465-71.

5. Ragin CC, Reshmi SC, Gollin SM. Mapping and analysis of HPV16 integration sites in a head and neck cancer cell line. Int J Cancer 2004;110:701-9.

\section{Bariatric Surgery for Morbid Obesity}

TO THE EDITOR: In his review of the surgical treatment of morbid obesity, DeMaria (May 24 issue) ${ }^{1}$ lists key components of the preoperative medical evaluation; these components include screening for diabetes, hypertension, coronary artery disease, sleep apnea, pulmonary hypertension, and musculoskeletal disease. However, the role of esophagogastroduodenoscopy in this evaluation should also be mentioned. Although its routine use remains controversial, ${ }^{2,3}$ there is evidence for recommending esophagogastroduodenoscopy with biopsy and assessment of samples for Helicobacter pylori in all patients planning to undergo bariatric surgery, even if they are asymptomatic. ${ }^{4-6}$ Several arguments provide support for this statement. First, there is a lack of correlation between symptoms and endoscopic findings. ${ }^{4,5}$ Second, in patients who undergo Roux-en-Y gastric bypass, the bypassed gastric and duodenal mucosa may no lon- ger be within the reach of the endoscope postoperatively, making it more difficult to treat lesions that could have been diagnosed preoperatively. ${ }^{5} \mathrm{Fi}-$ nally, routine esophagogastroduodenoscopy has been shown to have a high diagnostic yield and a relatively low cost. ${ }^{5}$

\section{Christoph Gasteyger, M.D.}

University of Copenhagen

DK-1958 Frederiksberg, Denmark

chga@life.ku.dk

1. DeMaria EJ. Bariatric surgery for morbid obesity. N Engl J Med 2007;356:2176-83.

2. Korenkov M, Sauerland $S$, Shah $S$, Junginger T. Is routine preoperative upper endoscopy in gastric banding patients really necessary? Obes Surg 2006;16:45-7.

3. Azagury D, Dumonceau JM, Morel P, Chassot G, Huber O. Preoperative work-up in asymptomatic patients undergoing Rouxen-Y gastric bypass: is endoscopy mandatory? Obes Surg 2006; 16:1304-11.

4. Csendes A, Burgos AM, Smok G, Beltran M. Endoscopic and histologic findings of the foregut in 426 patients with morbid obesity. Obes Surg 2007;17:28-34. 